\title{
Commercial Power Line Communication Adaptation for Avionic Applications
}

\author{
M. Brison ${ }^{1,2,4}$, M. Bensetti ${ }^{1}$, R. De Lacerda ${ }^{3}$, F. Vinci Dos Santos $^{4}$, C. Taurand ${ }^{2}$ \\ ${ }^{1}$ GeePs - Group of electrical engineering - Paris, UMR CNRS 8507, CentraleSupélec, Univ Paris-Sud, Sorbonne \\ Universités, UPMC Univ Paris 3-11, rue Joliot-Curie, Plateau de Moulon, 91192 Gif-sur-Yvette, France \\ 2 Thales Avionics, Hardware department, 25 Rue Jules Védrines, 26000 Valence, France \\ ${ }^{3}$ L2S Laboratoire de Signaux et Systèmes - UMR CNRS 8506, CentraleSupélec/Université Paris-Sud, 3 Rue \\ Joliot-Curie, 91192 Gif-sur-Yvette, France \\ ${ }^{4}$ Advanced Analog Design Group, CentraleSupélec, 3 Rue Joliot-Curie, 91192 Gif-sur-Yvette, France
}

\begin{abstract}
Aircraft designers believe that Power Line Communication (PLC) technology is a solution to reduce the number of cables aboard. One strategy to implement PLC networks in aircrafts is adapting commercial residential PLC solutions to the avionic environment. We propose in this paper a coupling architecture that improves PLC performance in terms of signal to noise ratio (SNR) when deployed on monofilar avionic networks. We also show that our proposal complies with the aeronautical electromagnetic standard (DO-160) through experimental validation. The proposed coupling architecture is investigated and validated through simulations and measurements. We analyze the performance of the PLC in terms of 3 parameters: S-parameters (transfer function), SNR and currents circulating on the aircraft circuit that must comply with the aeronautical electromagnetic regulations. The study also includes a real case scenario with the presence of a switch-mode power supply, which introduces distortion on the network and deteriorates the PLC performance. A comparison with a standard coupling circuit is performed and the results are promising.
\end{abstract}

\section{INTRODUCTION}

Aircraft designers are faced with the challenge of increasing the number of cables/connexions without increasing the weight of the aircraft. This increase is linked to the electrification of previously hydraulic or mechanical functions as well as the advent of new on-board features. Power Line Communication (PLC) appears as a solution to limit the proliferation of cables, given that the aircraft is an environment with strict electromagnetic interference (EMI) regulations that hamper the use of wireless communication technology. PLC is now a well-established communication technology for the Home Area Network (HAN) market. This type of technology uses the residential power distribution network (PDN) to ensure communication between devices. On the residential case, the network is built through the superposition of high-frequency signals on the $50 / 60 \mathrm{~Hz}$ network.

From the semiconductor industry perspective, the aircraft PLC market is too small to justify the conception of a new PLC standard. As a consequence, our efforts are geared to the implementation of an aeronautical PLC solution based on a HAN PLC chipset available on the market. Such a solution must comply with the EMI regulations and simultaneously support enough data throughput to ensure the communication among on-board equipments. This adaptation shall involve custom programming and hardware modifications, which are required due to physical differences between the aircraft and the residential power network.

In this paper, we focus on the adaptation of the coupling circuit (CC) for a residential broadband PLC modem with high data rate $(>10 \mathrm{MBps})$. The broadband PLC unit is an off-the-shelf product marketed for residential power networks. We propose a coupling architecture adapted to the aeronautical monofilar power network. We compare our solution with the provided HAN coupling circuit in terms of signal-to-noise ratio (SNR), with and without a Switched-Mode Power Supply (SMPS) unit connected. Emitted current measurements are performed on the cable to check the compliance of the solution with the avionic standard DO-160 [1].

\section{COUPLING A HAN PLC MODEM TO AN AVIONIC POWER NETWORK}

Typically, two types of electrical PDNs are deployed in an aircraft, a three-phase alternating current (AC) network $115 \mathrm{~V} / 400 \mathrm{~Hz}$ and a $28 \mathrm{~V}$ direct current (DC)network. We focus herein on the $28 \mathrm{~V}$ DC power line. The 28 DC network is deployed mostly for medium or low power loads and it is spread throughout the whole aircraft. This particular PDN is rarely cabled as a bifilar network. The power distribution unit is connected to the load by a single wire, so the return current goes through the fuselage of the aircraft. Hence, the fuselage is the electrical ground and/or the return current path for all electrical equipment aboard.

Studies on PLC to avionic power networks have already been published. One of the main endeavors that tackled this area is the "Transmissions in Aircraft on Unique Path Wires" (TAUPE) project, whose focus was to explore the general use of PLC technology in aircrafts. Within the TAUPE collaboration, commercial broadband PLC modems were used to perform EMC and communications tests [2], [3] and yielded encouraging results. However, these results were obtained over a bifilar network, which is not representative of the majority of 


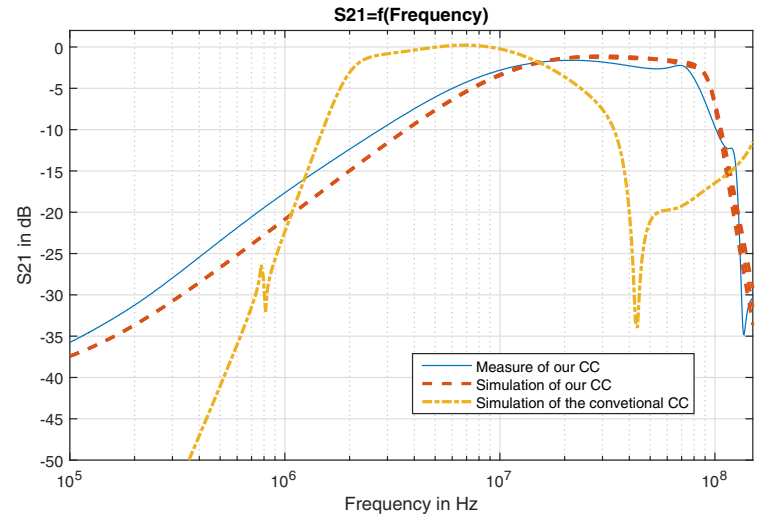

Fig. 1. S-parameter comparison between standard CC and our modified CC

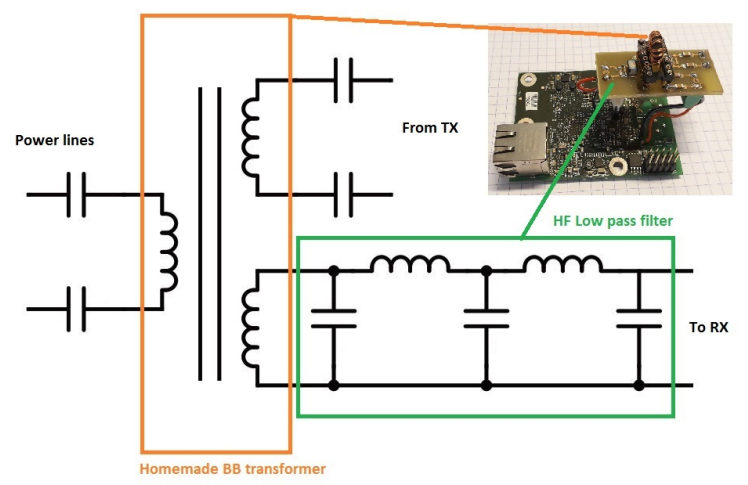

Fig. 2. Schematic and Photo of our $\mathrm{CC}$

avionics PDNs. More recently, PLC solutions have been employed for specific aircraft subsystems like actuators command [4] or motor control [5]. This work is complementary to these earlier efforts and aims to provide further information about the deployment of PLCs over monofilar networks, which is the most common PDN cabling.

From an electrical perspective, residential and aircraft PDNs can be equally modeled as transmission lines (TLs). The physical differences between both networks results on different TL characteristic impedances (Zc). Commercial PLC modems are designed to work on power-lines with a $\mathrm{Zc}$ of around $100 \Omega$. Avionics power-lines, configured as a single wire $5 \mathrm{~cm}$ above a ground plane representative of the 28VDC PDN, exhibit a $\mathrm{Zc}$ around $300 \Omega$ [6]. This impedance mismatch leads to additional loss and, as a consequence, communication degradation. A solution to improve the efficiency of the PLC link is to modify the coupling circuit of the PLC modem to be adapted to the avionic characteristic impedance.

\section{A. The Coupling Circuit (CC)}

The conventional coupling circuit is optimised to typical HAN PDN conditions. Depending on the cabling, it is necessary to adapt the coupling circuit to optimise the transfer

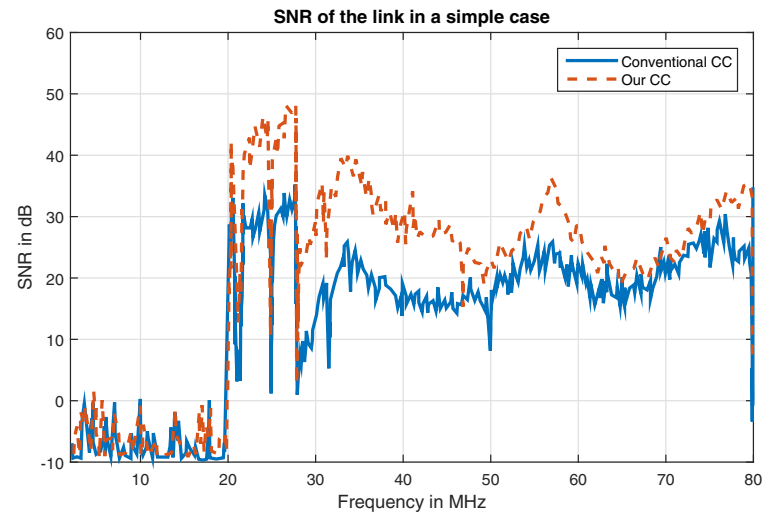

Fig. 3. SNR comparison between an old coupling circuit and the new one

function, which affects the communication quality between devices [5]-[7]. One of the solutions could be the use of an adaptive coupling circuit, but it is still a research challenge. For the narrowband PLC case, detailed design considerations of coupling is provided in [7]. The comparison between the differential and common mode coupling for the aerospace context is presented in [6], and on the three-phase avionic power network at [5].

In this work, we introduce the implementation and test of a modified coupling circuit based on the adaptation of the Sparameter using the G.hn standard. The G.hn standard uses the [1.8-86] $\mathrm{MHz}$ frequency band. Due to the high noise intensity generated by aircraft equipments on the frequency band below $20 \mathrm{MHz}$, we rely on the [20-86] $\mathrm{MHz}$ frequency band to establish communication. Our design objective is to set the Analog Front End (AFE) input impedance close to the characteristic impedance of the line over the upper frequency band to limit the impedance mismatch. To design the circuit, we employ a custom BB transformer, to reach the correct impedance matching, connected to the Tx ports and to the power lines with decoupling capacitors. A high frequency filter designed around the input impedance of the AFE chipset is connected between the BB transformer and the Rx ports.

A comparison of the transfer function of the conventional G.hn CC and our CC is presented in Fig.1. The conventional $\mathrm{CC}$ is the one used for the off-the-shelf G.hn PLC solution. We verify that the conventional $\mathrm{CC}$ is adapted to the [1.8-30] $\mathrm{MHz}$ frequency band but not for the whole [1.8-86] $\mathrm{MHz}$ band. As a consequence, it is not optimal for our avionic environment. We can observe that our CC is matched within the [20-86] $\mathrm{MHz}$ frequency band, as expected, and it provides an insertion loss of at most $-3 \mathrm{~dB}$. The schematic of our $\mathrm{CC}$ and a photo of the board with our CC are shown in Fig.2. We removed the original coupling circuit from the board to connect ours directly at the signal input/output of the AFE.

\section{B. Comparison between the two coupling circuits}

To verify the efficiency of the proposed coupling circuit, we compare the SNR perceived by the PLC modems with both 


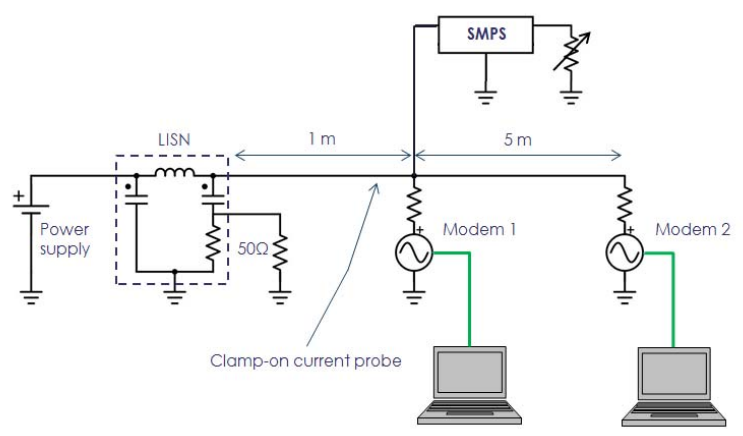

Fig. 4. Schematic of the test setup

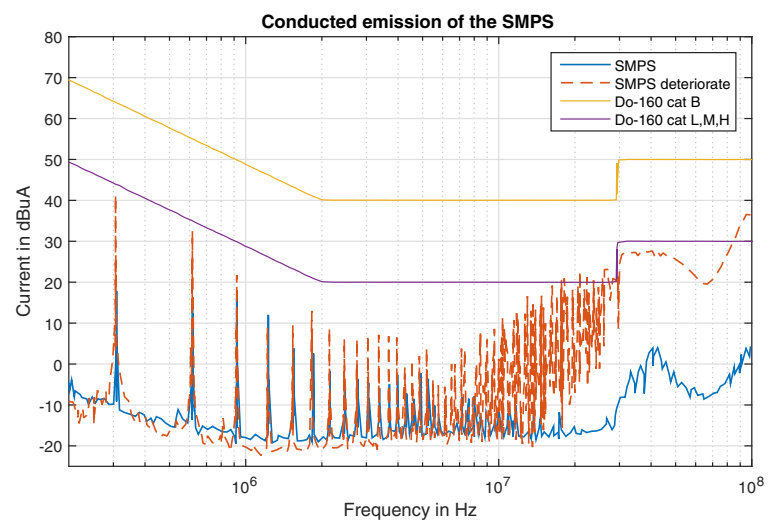

Fig. 5. Current emission of the SMPS in the two configurations

CCs. The test configuration is composed of two G.hn modems connected through a $10 \mathrm{~m}$ single wire $5 \mathrm{~cm}$ over a ground plane (as indicated on the DO-160).

We show in Fig. 3 the SNR of the link with the conventional $\mathrm{CC}$ in blue and with the improved $\mathrm{CC}$ in dotted red. The modified CC clearly improves the SNR , up to $20 \mathrm{~dB}$ at some frequency bands.

\section{EMI AND SNR MEASUREMENTS WITH A SMPS}

Our concept on the deployment of a avionic PLC is to include the PLC modems within the equipments to avoid designing a power supply for each modem. The equipment chosen in this case study is a 20W SMPS designed by Thales Avionics with a switching frequency of $150 \mathrm{kHz}$. This SMPS is set in two modes : a standard behaviour and a deteriorate behaviour. The deteriorate behaviour introduces higher perturbation levels, particularly present beyond $10 \mathrm{MHz}$. The EMI difference between the two modes reach up to $40 \mathrm{~dB} \mu \mathrm{A}$. The electromagnetic emissions of this equipment in these two configurations are shown in Fig. 5. The complete system, composed off the SMPS and the BB PLC modem, must comply with the standard DO-160 [1].

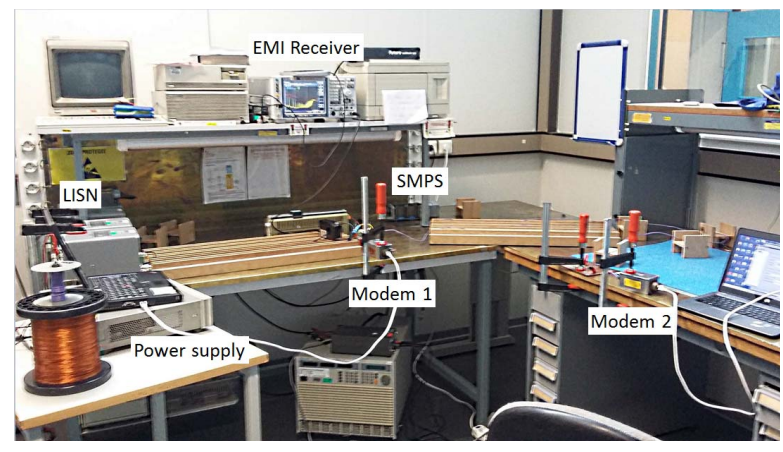

Fig. 6. Picture of the test setup

\section{A. Measurements Protocol}

Based on the DO-160, our measurement setup is described in Fig.4 and shown in Fig.6, These tests are done with both $\mathrm{CCs}$ in the two configurations of the SMPS (standard and deteriotate). The measurement procedure is the following:

- Switch on the power supply and wait until the modems finished the synchronization phase;

- Establish a real data transfer between the modems;

- Measure the average SNR provided by the modems;

- Measure cable currents with a spectrum analyser on MaxHold mode.

\section{B. Measurement Results}

In this section we present the measurements performed with both CCs on different configurations. The purpose of these results is to verify the performance of our $\mathrm{CC}$ and compare it with the conventional PLC HAN CC. The performance of our $\mathrm{CC}$ is presented in red dotted line and the conventional $\mathrm{CC}$ with the blue line. The PLC system uses an OFDM modulation where equally spaced subcarriers in frequency are used to carry the information. These modems use subcarriers that are $24.414 \mathrm{kHz}$ spaced to each others.

The first setup is set with the presence of the classic SMPS. Figs. 7 and 8 show the results respectively of the measured SNR and measured currents with both CCs setup. In terms of the SNR, we verify that our circuit outperforms the conventional CC in almost all frequencies in the [20-80] $\mathrm{MHz}$ band, providing gains above $20 \mathrm{~dB}$ in certain frequencies. In terms of currents, we observed that both setups are in conformity with the DO-160. However, as expected, the improvement of the communication link increased the currents between modems and our CC comes very close to the authorised levels within the [20-30] $\mathrm{MHz}$ band.

The second setup is set with the presence of the deteriorate SMPS, which is a worst case scenario in terms of noise distortion. Figs. 9 and 10 present the SNR and the EMI measurements, respectively. In this scenario, the modem with the conventional $\mathrm{CC}$ is not able to establish communication. SNR and EMI measurements are thus unavailable for this configuration. With our CC, the PLC modems establish communication achieving in some frequencies SNRs above $10 \mathrm{~dB}$. 


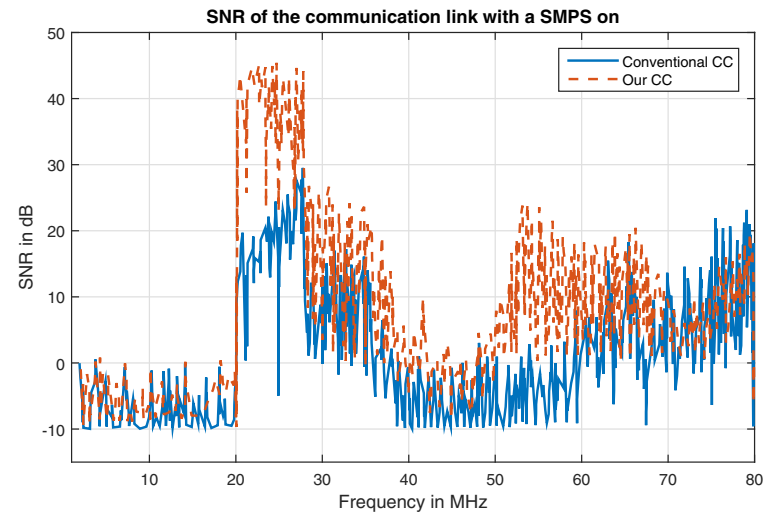

Fig. 7. SNR in the case SMPS + modem

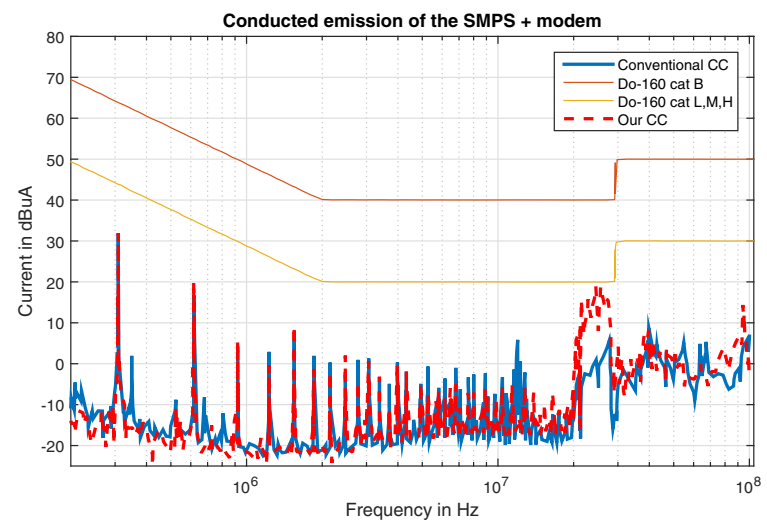

Fig. 8. Conducted emission SMPS+modem

However, the large noise generated by the deteriorate SMPS has an impact on the PLC performance and the data-rate is lower than the first setup. In terms of current emissions, the result shows that it does not comply with the DO-160 Category B but it complies with the others.

\section{CONCLUSions}

In this paper we demonstrate that coupling circuits used in commercial BB PLC systems are not well adapted to avionics 28V DC PDNs and we introduce a modified CC. Our modified CC was able to improve SNR and satisfy the Aeronautical EMI conditions to most of the cases. We have demonstrated that the concept of adding broadband PLC modems to SMPS equipment are feasible, even for worst case of noise emissions. Moreover, SNR and EMC measurements proved that communication is possible while complying to current emission limits specified in the DO-160, when our proposed CC is used.

\section{REFERENCES}

[1] Environmental conditions and Test procedures for airborne equipment, DO-160, RTCA DO-160, 2010.

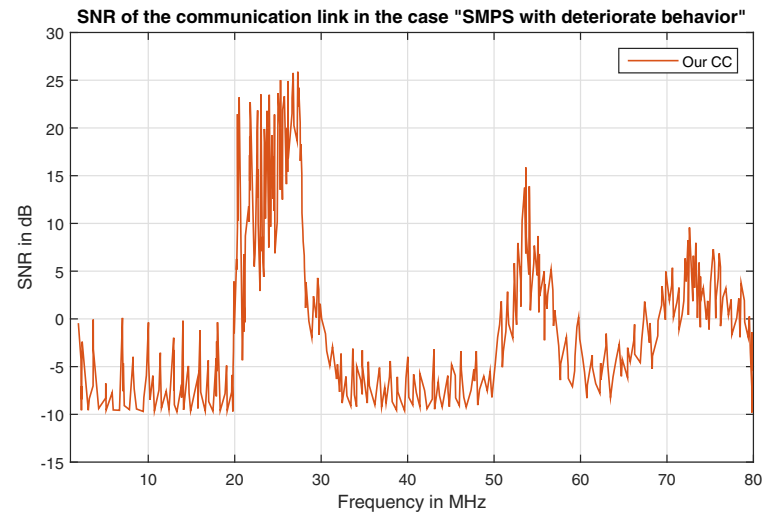

Fig. 9. SNR of the PLC link with the degraded SMPS

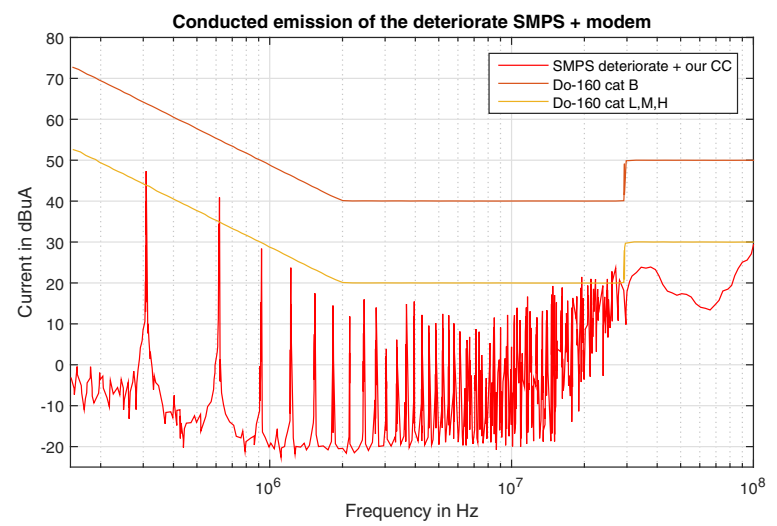

Fig. 10. Conducted emission of the degraded SMPS + BB PLC modem

[2] S. Dominiak, H. Widmer, M. Bittner, and U. Dersch, "A bifilar approach to power and data transmission over common wires in aircraft," in Digital Avionics Systems Conference (DASC), 2011 IEEE/AIAA 30th, Oct 2011, pp. 7B4-1-7B4-13.

[3] S. Dominiak, G. Vos, T. ter Meer, and H. Widmer, "Achieving emc emissions compliance for an aeronautics power line communications system," in Aerospace EMC, 2012 Proceedings ESA Workshop on, May 2012, pp. 1-6.

[4] T. Larhzaoui, F. Nouvel, J.-Y. Baudais, P. Degauque, and V. Degardin, "Ofdm plc transmission for aircraft flight control system," in Power Line Communications and its Applications (ISPLC), 2014 18th IEEE International Symposium on, March 2014, pp. 220-225.

[5] K. Kilani, "Feasibility of powerline communication on a three-phase cable between pwm inverter and motor for avionics applications," Ph.D. dissertation, University Lille 1, 2013.

[6] F. Grassi, S. Pignari, and J. Wolf, "Channel characterization and emc assessment of a plc system for spacecraft dc differential power buses," Electromagnetic Compatibility, IEEE Transactions on, vol. 53, no. 3, pp. 664-675, Aug 2011.

[7] P. J. Van Rensburg and H. C. Ferreira, "Step-by-step design of a coupling circuit with bi-directional transmission capabilities," Proceedings of the 8th International Symposium on Power-Line Communications and its Applications, pp. 238-243, 2004. 